Please do not remove this page

RMIT

UNIVERSITY

\title{
An analysis of the thermodynamic conditions for solid powder particles spreading over liquid surface
}

Nguyen, Thanh; Eshtiaghi, Nicky; Hapgood, Karen; Shen, Wei

https://researchrepository.rmit.edu.au/esploro/outputs/9921857664901341/filesAndLinks?institution=61RMIT_INST\&index=null

Nguyen, T., Eshtiaghi, N., Hapgood, K., \& Shen, W. (2010). An analysis of the thermodynamic conditions for solid powder particles spreading over liquid surface. Powder Technology, 201(3), 306-310.

https://doi.org/10.1016/j.powtec.2010.04.019

Document Version: Submitted Version

Published Version: https://doi.org/10.1016/j.powtec.2010.04.019

Repository homepage: https://researchrepository.rmit.edu.au

(c) 2010 Elsevier B.V. All rights reserved.

Downloaded On 2023/04/26 15:48:11 +1000 
Citation:

Nguyen, T, Eshtiaghi, N, Hapgood, K and Shen, W 2010, 'An analysis of the thermodynamic conditions for solid powder particles spreading over liquid surface', Powder Technology, vol. 201, pp. 306-310.

\title{
An analysis of the thermodynamic conditions for solid powder particles spreading over liquid surface
}

\author{
Thanh H. Nguyen ${ }^{\mathrm{a}, \mathrm{b}}$, Nicky Eshtiaghi ${ }^{\mathrm{b}}$, Karen P. Hapgood ${ }^{\mathrm{b}}$ and Wei Shen ${ }^{\mathrm{a}^{*}}$ \\ ${ }^{a}$ Australian Pulp and Paper Institute, Department of Chemical Engineering, Monash \\ University, Melbourne, Victoria, Australia, 3800 \\ ${ }^{b}$ Monash Advanced Particle Engineering Laboratory, Department of Chemical \\ Engineering, Monash University, Melbourne, Victoria, Australia, 3800 \\ Email addresses of authors: Thanh. Nguyen (thanh.h.nguyen@eng.monash.edu.au), \\ Nicky Eshtiaghi (nicky.eshtiaghi@eng.monash.edu.au), Karen Hapgood \\ (karen.hapgood@eng.monash.edu.au),Wei Shen (wei.shen@eng.monash.edu.au) \\ *Corresponding Author. Tel: +61 39905 3447; Fax: +61 39905 3413; \\ Email: wei.shen@eng.monash.edu.au (W. Shen) \\ Postal address: Department of Chemical Engineering, Faculty of Engineering, \\ Monash University, Clayton Campus, Wellington Road, Clayton 3800, Victoria, \\ Australia
}

\begin{abstract}
The spreading of solid powder over a liquid surface is a prevalent phenomenon encountered in many industrial processes such as food and pharmaceutical processes. The driving force for powder spreading over a liquid surface is not clearly understood. The Marangoni effect due to a temperature gradient and the spreading coefficient for solid powder over liquid $\left(\lambda_{S / L}\right)$ have both been proposed as causes for powder spreading over liquids. The proposed $\lambda_{S / L}$ was based on the same form of the spreading coefficient for a liquid over a solid surface $\left(\lambda_{L / S}\right)$. Whereas $\lambda_{L / S}$ has a clear thermodynamic definition, the spreading coefficient of solid powder over liquid, $\lambda_{S / L}$, which was defined by simply interchanging the subscripts of the interfacial energy terms, has not been thoroughly analysed. Our experimental results showed that the spreading behaviour of solid powders over liquids cannot be explained or predicted by $\lambda_{S / L}$. In this study we focus on problems associated with the $\lambda_{S / L}$. Through a thermodynamic analysis we conclude that the existing parameter $\lambda_{S / L}$ is unable to predict the spreading behaviour of solid powder on liquid surface, since the interfacial energy approach does not capture the actual physical process of powder spreading over liquid surface. A closer examination of the powder spreading process reveals the
\end{abstract}


fundamental different between liquid spreading over solid surface and solid powder spreading over liquid. This work shows that further research is required to identify and analyse the physical mechanisms which are responsible for powder particles spreading over liquid surfaces.

Keywords: Liquid marbles, powder spreading over liquid, thermodynamic condition, spreading coefficient.

\section{Introduction}

The spreading of liquids over solid surfaces is one of the most widely encountered phenomena in our daily life and also in technology. Its applications in technology range from laundry, lubrication, dyeing/printing to the pharmaceutical and food industries. Thermodynamic predictions of liquid spreading over solid surfaces have been developed for many of these applications [1]. The opposite phenomenon, i.e. solid powder spreading over a liquid surface, is also seen in our daily life and in technology; this phenomenon has important implications in wet granulation [2-4] and food processing [5] and has attracted a number of investigations on the prediction of conditions under which solid powder can spread over a liquid surface.

In many cases when a drop of aqueous liquid is placed on a bed of hydrophobic powder, the powder particles do not spontaneously spread over the drop surface. Instead, the liquid drop must be allowed to roll over the bed and pick up powder particles by contact to gain a full powder coverage and to form a liquid marble [6]. McEleney et al. [7] showed that hydrophobised fine metal powders do not spread over water sessile drops unless the drops are allowed to roll over the powder bed. The same behaviour is seen for PTFE powder where the powder particles do not spread over water sessile drop (Figure 1).

However, there have been observations where hydrophobic powders spread over liquid sessile drops when the drops were placed on the powder bed gently without any rolling movement on the bed. In our laboratory, we observed that salicylic acid crystals can spread over sessile water drops (Figure 2). The spreading tendency of salicylic acid crystals over water was found to be highly sensitive to minute external 
disturbance. Slight kinetic energy $(0.5 \mathrm{~cm}$ impact height) of the water droplet can result in the crystals spreading over the water droplet.

Some published work explained the observed "spontaneous" powder spreading behaviour over liquid drops using the concept of favourable thermodynamic spreading coefficients [8]. The spreading coefficient criterion, which is based on the interfacial energetics of the solid-liquid interface, does not offer reliable prediction to the powder behaviours over liquid [9]. While it is obvious that phenomenon of powder spreading over liquid requires more extensive investigations, the purpose of this study is to provide a simple analysis of the spreading coefficient model by Rowe [8] and explain the problems associated with the original development of the model. The proposed spreading coefficient of solid over liquid $\left(\lambda_{S / L}\right)$ was derived to predict the spreading behaviour of powder over a liquid. The derivation of this model used an analogy of the thermodynamic spreading coefficient for a liquid spreading over a solid surface (which is defined as $\lambda_{L / S}=\gamma_{S V}-\gamma_{L V}-\gamma_{S L}[1]$, or $\lambda_{L / S}=W_{A(L / S)}-W_{C(L)}$ (see the following section)). The calculation of $\lambda_{S / L}$ further considers the work of adhesion due to the polar and non-polar intermolecular interactions [10] as follows [8]:

$$
\lambda_{S / L}=4\left[\frac{\gamma_{S}^{d} \gamma_{L}^{d}}{\gamma_{S}^{d}+\gamma_{L}^{d}}+\frac{\gamma_{S}^{p} \gamma_{L}^{p}}{\gamma_{S}^{p}+\gamma_{L}^{p}}\right]-2 \gamma_{S}
$$

It should be pointed out that although the derivation of equation (1) takes the form of the thermodynamic spreading coefficient, the intermolecular interactions described by Wu's approach [10] is empirical and their thermodynamic validity has not been proven. This criterion has been used by other authors to predict powder spreading over liquid [3, 11]. However, the physical validity of the criterion has not been thoroughly analysed in previous research. If the literature surface tension values for PTFE and water are substituted into Equation (1), a positive value for $\lambda_{S / L}$ is obtained, suggesting that the spreading of PTFE over water would occur, whereas in fact the PTFE powder does not spread over water droplet at all [9]. The predictions made using the $\lambda_{S / L}$ model do not agree with the experimental observations.

The thermodynamic criterion for liquids to spread on solid surfaces has been well 
studied [1]. The spreading coefficient for liquid spreading over a solid $\left(\lambda_{L / S}\right)$ provides a thermodynamic prediction of whether such a process can occur spontaneously. The spreading of solid powder over a liquid surface, however, is a very different phenomenon compared to liquid spreading over solid. When a solid powder aggregate expands its apparent coverage over a liquid surface, the behaviour of the molecules in the powder particles is different from the behaviour of molecules of the liquid as they spread over a solid surface. For a hydrophobic powder, solid powder particles do not increase their surface area as they spread. Instead, powder aggregates merely disintegrate as they move towards the free liquid surface. The only change in this process is that a fraction of the solid powder surface becomes a solid/liquid interface. This critical difference between the two spreading processes has not been addressed in sufficient detail previously. The model of solid powder spreading over a liquid surface using $\lambda_{S / L}$ assumes these two spreading processes are similar and can be described by similar physics. It is necessary that the thermodynamics of the process of solid powder aggregate expansion over a liquid surface be studied in detail to allow a clear understanding of the free energy changes of the process and the thermodynamic implications. This analysis will verify the validity of the spreading coefficient $\lambda_{S / L}$ as a criterion for the prediction of powder spreading over a liquid.

To do so we focus on changes in the solid and liquid interface before and after powder spreading over the liquid surface and clarify the correlations between these changes with the free energy changes of the surfaces and interface. Our analysis shows that the existing spreading coefficient is not valid and unable to predict the spreading of solid powder over liquid. Experiments were also presented to support this view.

\section{Experimental}

To observe the capacity for a powder to spread over a liquid surface, salicylic acid (Sigma-Aldrich, particle size distribution from $90 \mu \mathrm{m}$ to $2 \mathrm{~mm}$ ) and polytetrafluoroethylene (PTFE, Sigma-Aldrich, average particle size $=100 \mu \mathrm{m}$ ) were used as the hydrophobic powders for solid spreading. Distilled water was used as the bulk liquid phase. In experiments where powder spreads over liquid surface, Steven's method [5] was employed to observe the behaviour of the powder as it impacts on the liquid surface. In each experiment, approximately 0.15 to $0.25 \mathrm{~mL}$ of powder was 
deposited on a Petri dish of water with the impact height kept constant at $2 \mathrm{~cm}$. The powder disintegration and spreading on the liquid surface was then captured using a digital camera. In experiments where liquid droplets were deposited on powder bed, water droplets were released onto the powder bed from a height of $0.5 \mathrm{~cm}$. The droplet surface coverage by the powders was examined. The degree of droplet surface coverage can be visually and unambiguously assessed when the images of the drops were captured using an optical microscope (Motic 2300 moticam) at a magnification of 11.25 times. The droplet volume was approximately $13 \mu \mathrm{L}$.

\section{Results and Discussion}

\subsection{Spreading coefficient and its thermodynamic foundation - liquid spreading over solid substrate}

The spreading coefficient for a liquid over a solid or another immiscible liquid surface is used to predict whether or not the spreading of a liquid on either a solid surface or an immiscible liquid surface is spontaneous. The spreading coefficient for a liquid over a solid surface is thermodynamically defined as:

$$
\lambda_{L / S}=\gamma_{S}-\gamma_{L}-\gamma_{S L}
$$

where $\gamma_{S}, \gamma_{L}$ and $\gamma_{S L}$ are the interfacial energies of the solid, liquid and solid-liquid interfaces involved. The spreading coefficient in Equation (2) is also applicable to the spreading of a liquid over another immiscible and denser liquid when the subscripts are properly changed [1]. The spreading coefficient can also be written in the following form:

$$
\lambda_{L / S}=W_{A(S L)}-W_{C(L)}
$$

$W_{A}$ and $W_{C}$ are the work of adhesion between liquid and solid and work of cohesion of the liquid. If $W_{A}$ is greater than $W_{C}$ (i.e. $\lambda_{L / S}>0$ ), the liquid has a stronger tendency to increase its contact with the solid than to reduce its contact area with the solid; the liquid will therefore spread on the solid surface spontaneously. Conversely, if $W_{C}$ is greater than $W_{A}$ (i.e. $\lambda_{L / S}<0$ ) the liquid will have a stronger tendency to reduce its 
contact area with the solid and spontaneous spreading of the liquid over the solid will not occur.

Whilst the thermodynamic definition of liquid spreading over a solid surface is clear, it is still beneficial to carefully observe the physics of the spreading process and understand the behaviour of the liquid molecules in the spreading process. When a liquid phase spreads over a solid or another immiscible and denser liquid, the spreading liquid increases its surface area as well as its interfacial area with the substrate phase. Molecules of the spreading liquid will have to move from the bulk onto the freshly formed surface and interface with the substrate phase. This increase in surface area is against the natural trend of liquids assuming the smallest surface area. The reason for this to occur is that the surface tension of the substrate phase is greater than the sum of the surface tension of the spreading liquid and the interfacial tension of the spreading and substrate phase. Alternatively, from a surface free energy point of view, the work of adhesion between the spreading liquid and the substrate phase is greater than the work of cohesion of the spreading liquid.

The spreading of a liquid over a solid surface bears thermodynamic similarity to the spreading of a liquid over another, but immiscible, liquid. This similarity allows us to appreciate the spreading coefficient through the classic experiment performed by Franklin which clearly demonstrated the behaviour of molecules of the spreading liquid. In 1774, Franklin investigated the effect of oil on the tranquillity of the water surface [12]. Upon arriving at a pond on a windy day, Franklin deposited a teaspoon of olive oil onto the water surface in the pond and observed the formation of an oil film that covered approximately half an acre on the pond surface. This oil layer acted as lubricant against the wind, preventing waves being created on the pond surface. The oil film appeared to be very thin and work carried out by Lord Rayleigh showed that the film of the olive oil on the water was one molecular thick (approximately 25 $\AA$ ) [12]. A schematic diagram of the oil droplet before and after spreading is shown in Figure 3.

The spontaneous increase in the oil/water interface forces the oil surface area to increase. This process brings oil molecules from the bulk of the drop onto the 
interface and the surface. A thermodynamic derivation taking the initial and final states may be presented as follows:

$$
\Delta G=A \gamma_{W O}+A \gamma_{O}-A \gamma_{W}-a \gamma_{O}
$$

Where $A$ is the surface area of the pond, $a$ is the surface area of the teaspoonful of oil before it was deposited onto the water surface. Since $A \gg>a$, this equation can be written as

$$
\frac{\Delta G}{A}=\gamma_{W O}-\gamma_{W}+\gamma_{O}=-\lambda_{O / W}
$$

This derivation leads to the following equation,

$$
\lambda_{O / W}=W_{W O}-W_{O}
$$

Substituting literature values of surface tension of olive oil $(32.5 \mathrm{mN} / \mathrm{m}$,$) [13] and$ interfacial tension of olive oil and water $(23.6 \mathrm{mN} / \mathrm{m})$ [14] into Equation (5) leads to a positive $\lambda_{O / W}(15.9 \mathrm{mN} / \mathrm{m})$ which predicts that oil will spread over water. This result agrees with Franklin's experiment.

\section{2. "Spreading coefficient of solid particles over liquid surface"}

Figure 4 shows the schematic of a small solid particle aggregate as it disintegrates and expands over liquid surface. We assume that solid particles are inert and do not dissolve in the liquid. The total surface area of the particles in the aggregate is much greater than the apparent outmost surface area defined by volume and the shape of the aggregate (Figure 4). This situation is very different to the teaspoonful of oil discussed above, where the surface area of the teaspoonful of oil equals exactly to the surface area of the volume that defines it (Figure 3).

When powder aggregates disintegrate and expand over the liquid surface, a fraction of the powder surface will be in contact with the liquid, establishing a solid/liquid interface. The other fraction will remain unchanged (Figure 4). A solid particle will 
establish a solid/liquid area larger than the liquid area it displaces, depending on the wetting condition of the solid particle by the liquid as schematically shown in the simplified schematic diagram in Figure 5. The ratio of the solid/liquid interface area and the liquid surface area it replaces (see Figure 5) is denoted by $\beta$. Under the assumption that solid particles are spherical,

$$
\beta=\left[1+(h / r)^{2}\right]
$$

If the solid particle is not wettable by the liquid (i.e. contact angle $\theta>90^{\circ}$ ), $\beta$ would be in the range between 1 and 2. However, if surface roughness of the particles is considered, $\beta$ will be larger than 1 but less than 2 , due to the Cassie-Baxter effect [15].

As the powder aggregates try to disintegrate, expand and cover the liquid surface, the inter-particle attraction forces must be overcome so that more solid particles can move and establish more particle/liquid interfaces. Since solid powder particles of micron size or larger [7] have very limited intimate contact at the molecular scale between them, the short range attraction forces between the particles due to the van der Waals interactions are therefore weak [16], but they are responsible for holding the particles in the aggregates together. However, the inter-particle attraction forces bear no quantitative relationship to the solid surface free energy and cannot be described by the solid surface free energy. It is therefore incorrect to use the surface energy of the solid to define the work of cohesion between solid particles, such as in Equation (1).

If the powder spreading process over a liquid (Figure 4) is examined from a thermodynamic viewpoint, the initial and final states need to be defined. With the following assumptions, Figure 4 provides the initial and final states of the spreading process:

- The solid particles are hydrophobic and will not be fully wetted by the liquid and remain floating on liquid surface.

- The surface area of the particles does not change after contacting water.

- The surface tension of the liquid does not change when exposed to solid powder. 
A liquid surface coverage fraction $\Phi$ is used to represent the fraction of the liquid surface that becomes covered by the solid powder after the powder spreads over the liquid surface. The total surface free energy of the initial state is $\left(\gamma_{S} A_{S}+\gamma_{L} A_{L}\right)$, and the total surface free energy of the final state is $\left(\gamma_{L}[1-\Phi] A_{\mathrm{L}}+(1+\beta) \Phi A_{L} \gamma_{L S}+\left[A_{S^{-}}(1+\beta) \Phi\right.\right.$ $\left.A_{L}\right] \gamma_{S}$ ). The free energy change of the powder spreading process will then be:

$$
\begin{aligned}
& \Delta G=-A_{L} \Phi W_{A(L S)}-A_{L} \beta \Phi\left(\gamma_{S}-\gamma_{L S}\right) \\
& \frac{\Delta G}{\Phi A_{L}}=-W_{A(L S)}-\beta\left(\gamma_{S}-\gamma_{L S}\right)
\end{aligned}
$$

where $\beta$ is defined in Figure 5. Equation (8) describes the free energy change between the final and initial states of the solid particle aggregates disintegrate and cover the liquid surface. Equation (9) further shows that only a fraction $(\Phi)$ of the liquid surface replaced by solid/liquid interface was responsible for free energy change. It is not possible for the thermodynamic relationship of Equation (1) to be derived from Equations (8) and (9). This result indicates that the original considerations for the "spreading coefficient of solid over liquid $\left(\lambda_{S / L}\right)$ " does not capture the physical process of powder spreading over a liquid phase and therefore is an incorrect parameter for predicting powder spreading over liquid surfaces.

\subsection{Driving forces for solid particles to spread over liquid surface}

McEleney et al. [7] has recently suggested that the Maragoni effect due to temperature gradient on the liquid drop surface may be a driving force causing the spreading of solid particles over liquid surface. Their suggestion was based on the possible heating effect of the microscope light source on the north pole of the liquid drop under investigation. Under this assumption, the temperature difference between the north and the south poles of the liquid drop could cause circulation the liquid over the drop surface. Whilst the Maragoni effect may be one of the possible driving forces causing powder spreading, it is possible that there may other interfacial forces that are responsible for this phenomenon. More extensive research is required to identify and analyse the physical and physicochemical mechanisms which generate the driving forces and cause powder particles to spread over liquid surfaces. 


\section{Conclusions}

This study analysed and compared the spreading processes of liquid over a solid (or liquid over an immiscible liquid) and to solid particle aggregates over a liquid. These two processes are fundamentally different in their physical nature. Thermodynamic analysis of the two different spreading processes showed that the "spreading coefficient of solid over liquid" proposed previously [8] is invalid, as it does not capture the actual physical process. Other factors, including the Marangoni effect due to temperature and liquid surface tension gradients, are likely to be the more dominant driving forces which cause the initial movement of solid particles over the liquid surface. Further research efforts are required to identify and analyse powder spreading driving forces.

\section{Acknowledgements}

This project was supported financially by the Australian Research Council Discovery program (DP0770462), and N. Eshtiaghi's scholarship was funded by the Monash Graduate Research School.

\section{References}

1. Adamson, A.W. and A.P. Gast, Physical Chemistry of Surfaces. 1997. 6th ed, New York, USA: John Wiley \& Sons. p. 466

2. Planinsek, O., et al., 2000. The utilization of surface free-energy parameters for the selection of a suitable binder in fluidized bed granulation. International Journal of Pharmaceutics, 207(1-2): p. 77-88.

3. Tuske, Z., et al., 2005. The role of the surface free energy in the selection of a suitable excipient in the course of a wet-granulation method. Powder Technology, 155(2): p. 139-144.

4. Zajic, L. and G. Buckton, 1990. The use of surface energy values to predict optimum binder selection for granulations. International Journal of Pharmaceutics, 59(2): p. 155-164.

5. Galet, L., et al., 2004. The Wetting Behaviour and Dispersion Rate of Cocoa Powder in Water. Food and Bioproducts Processing, 82(4): p. 298-303.

6. Aussillous, P. and D. Quere, 2001. Liquid marbles. Nature, 411(6840): p. 924927.

7. McEleney, P., et al., 2009. Liquid marble formation using hydrophobic powders. Chemical Engineering Journal, 147(2-3): p. 373-382. 
8. Rowe, R.C., 1989. Surface free energy and polarity effects in the granulation of a model system. International Journal of Pharmaceutics, 53(1): p. 75-78.

9. Eshtiaghi, N., et al., 2009. Liquid marble formation: Spreading coefficients or kinetic energy? Powder Technology, 196(2): p. 126-132.

10. Wu, S., 1973. Polar and Nonpolar Interactions in Adhesion. The Journal of Adhesion, 5(1): p. 39 - 55-39 - 55.

11. He, X., et al., 2008. Development of a rapidly dispersing tablet of a poorly wettable compound--formulation DOE and mechanistic study of effect of formulation excipients on wetting of celecoxib. International Journal of Pharmaceutics, 353(1-2): p. 176-186.

12. Erbil, H.Y., Surface chemistry of solid and liquid interfaces. 2006. ed: WileyBlackwell.

13. Liu, H.-S., W.-C. Chiung, and Y.-C. Wang, 1994. Effect of lard oil, olive oil and castor oil on oxygen transfer in an agitated fermentor. Biotechnology Techniques, 8(1): p. 17-20.

14. Fisher, L.R., E.E. Mitchell, and N.S. Parker, 1985. Interfacial Tensions of Commercial Vegetable Oils with Water. Journal of Food Science, 50(4): p. 1201-1202.

15. Cassie, A.B.D. and S. Baxter, 1944. Wettability of Porous Surfaces. Transactions of the Faraday Society, 40: p. 546-551.

16. Kendall, K., Molecular Adhesion and its Applications. 2001. ed, New York: Kluwer Academic. 


\section{Figure Captions}

Figure 1. Non-spreading behaviour of PTFE powder on a gently deposited water droplet.

Figure 2. Surface coverage from a gently placed distilled water droplet (blue dye added as visual aid) on microcrystalline salicylic acid powder bed.

Figure 3. Schematic diagram of Franklin's experiment of oil spreading on water.

Figure 4: A schematic of a solid powder aggregate disintegrates and expands over a liquid surface.

Figure 5: A non-wettable powder particle contacting a liquid surface. The area of the solid/liquid interface (broken line) and the area of the liquid surface (solid line) the particle replaces as it spreads on the liquid surface; these areas are not the same. The ratio of solid/liquid interface (broken line) to liquid surface (solid line) is represented by $\beta$ in equation (7). 


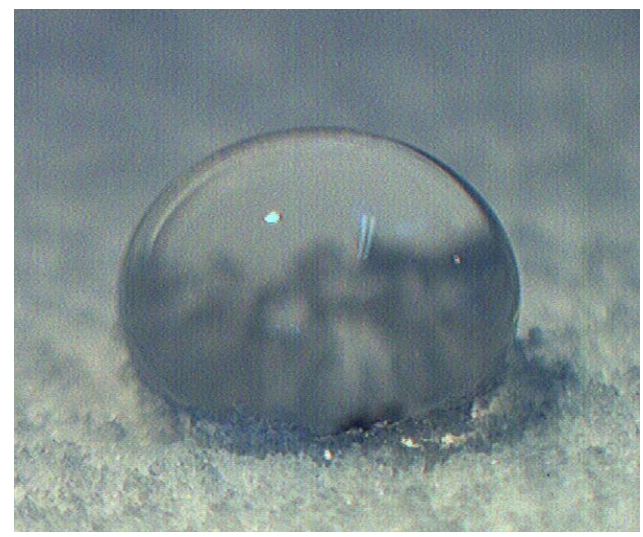

Figure 1

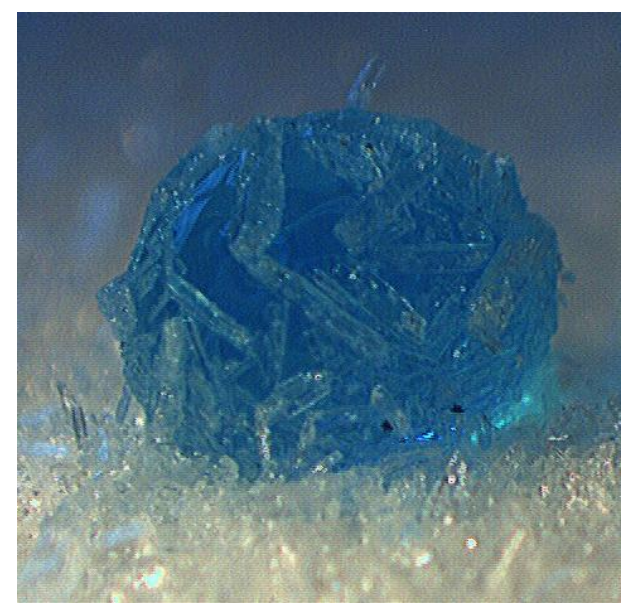

Figure 2 


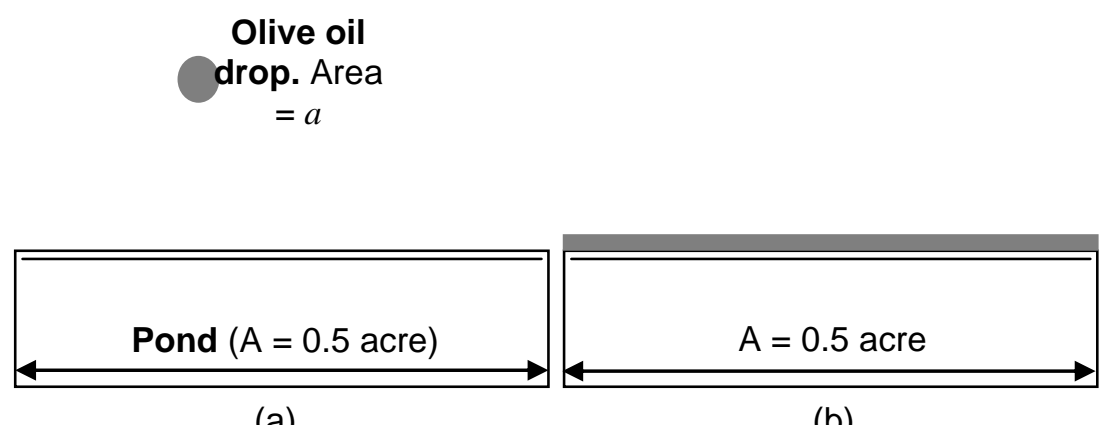

(a)

(b)

Figure 3

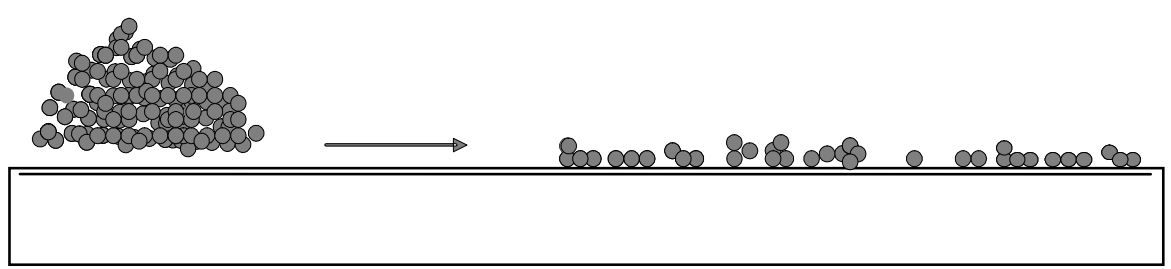

Figure 4 


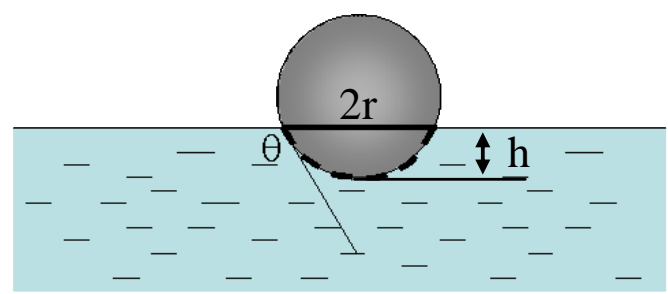

Figure 5

Page 15 of 15 